\title{
EDITORIAL
}

\section{Structural imaging in the clinical diagnosis of Alzheimer's disease: problems and tools}

The techniques of CT and MRI are the most commonly used instrumental aids in the diagnosis of dementia and Alzheimer's disease. Their use is often uncritical or suboptimal as to when the examination should be prescribed, which of the two techniques should be used, how much information can be obtained, and how often the information gives significant help in the differential diagnostic process. This claim is supported by the discrepancy between clinical practice-where imaging is generously used - and evidence based guidelines advising that imaging is not needed except in a few particularly uncertain cases. ${ }^{1}$

New imaging tools are emerging ${ }^{2}$ that might significantly change the way we presently use structural CT and MRI, but full mastery of current standard techniques is mandatory for the clinical neurologist and psychiatrist for at least two reasons. Firstly, the new tools might not be available for routine clinical work for years to come, and the challenges of improved differential and preclinical diagnosis will for the time being need to be met with traditional tools. Secondly, when new expensive technology will be available, physicians will need to place it in the proper clinical context of cost-effectiveness. The increasing gap between reduced financial resources on the one hand and the increasing availability of expensive diagnostic options on the other ${ }^{3}$ will make harder the task of estimating in each patient whether the large additional cost of new technology might be justified by the expected additional clinical information. In doing this, cost-effectiveness studies will probably not be available as supports, ${ }^{4}$ and decisions will rely only on the physician's own clinical skill and expertise.

Lastly, the increasing interest in the preclinical stages of dementia-be it Alzheimer's disease ${ }^{5}$ or vascular dementia ${ }^{67}$-will probably make imaging even more relevant in the near future. In these cases, the clinical findings are less clearcut than in overt dementia and objective disease markers might have greater weight in the diagnostic and prognostic judgement.

The aim of this editorial is to discuss the rationale and evidence behind present imaging practices in the diagnosis of Alzheimer's disease, highlight the cogent clinical questions that imaging might help to answer, review the available tools that could be used to extract more useful information from imaging examinations, and suggest avenues for future research. Particular attention is devoted to the key factors for such tools to be implemented in the clinical routine - that is, feasibility, added diagnostic value, and detection of early cases.

\section{Epidemiology of imaging practices for Alzheimer's disease}

On the basis of incidence data of dementia, ${ }^{8}$ in the European Union (population of about 380 million) between 5.5 and 8.9 million persons each year are eligible for structural imaging. If cases of incident mild cognitive impairment are also considered, the figure might be three times as high. ${ }^{9}$ Estimating the proportion of these patients who actually undergo structural imaging in Europe is a difficult task. A European agency directing the use of technology in medicine has been advocated but it is not yet active, ${ }^{10}$ and epidemiological data have never been collected on this issue. However, it is likely that the practice reflects what was suggested in 1994 by Rossor - that is, that "in elderly demented patients routine neuroimaging (with CT or MR) is the ideal that should be sought". ${ }^{11}$ This view is implicitly endorsed by some European national health systems, which refund the cost of one structural imaging examination during the course of a dementing disorder. ${ }^{12}$

Likewise, there are no data on which is more often carried out-CT or MRI-although the cost varies about threefold in most countries. It is my opinion that MRI is more often used in northern and central Europe, and CT in the Mediterranean countries. Which is preferred is often due more to local practical factors (availability, accessibility, etc) rather than to clinical and scientific considerations.

Although the agreement among different raters is known to be poor, ${ }^{13-15}$ in routine clinical settings the traditional reading technique of unstandardised subjective visual assessment by an expert rater is used. In the differential diagnosis of dementing disorders, this has three main aims: detecting reversible or treatable intracranial causes of cognitive impairment, detecting cerebrovascular disease, and detecting atrophic changes.

\section{Reversible or treatable intracranial causes of} cognitive impairment: is imaging necessary? Chronic subdural haematoma, tumour, and normal pressure hydrocephalus are known to cause cognitive impairment that can be mistaken for Alzheimer's disease or other primary dementias and can be easily demonstrated by CT and MR. Such conditions are severely disabling and fatal if untreated, but reversible after appropriate treatment. The possibility of missing some of these cases probably plays a part in the decision of physicians to scan most patients with cognitive impairment; this is also the case in other conditions that are, albeit infrequently, associated with fatal outcomes, such as minor head injury. ${ }^{16}$ Because some signs and symptoms often accompany reversible or treatable intracranial conditions, the question arises as to whether these can be used to identify patients eligible for scanning, thus saving a huge number of scanning procedures. ${ }^{16}$

Chui and Zhang estimated the yield of examinations used for the differential diagnosis of dementia in an Alzheimer's disease referral centre in the United States and found that the use of four clinical indicators (symptom onset before 60 years, non-insidious course, focal signs or symptoms, and gait disturbance) would have reduced the frequency of imaging studies by $33 \%$, missing information that would have significanly changed management in only one (false negative) case. ${ }^{17}$ In this patient, the finding of ventricular enlargement disproportionate to the degree of sulcal enlargement changed the diagnosis from "probable Alzheimer's disease" to "possible Alzheimer's disease or 
possible normal pressure hydrocephalus". However, it should be noted that the relatively high sensitivity of the clinical indicators found in the study of Chui and Zhang (95\%) might be inflated by the peculiar characteristics of their patients. As well as being relatively young (70 (SD 8) years), it is known that patients coming for observation at specialist centres may not be representative of the general population of patients with dementia and Alzheimer's disease, in that they are more educated, and have higher socioeconomic status ${ }^{18}$ and better physical health. The prevalence of at least three of the four clinical indicators (non-insidious course, focal signs or symptoms, and gait disturbance) might be more frequent in a less selected population of older patients due to higher frequency of cerebrovascular and other physical diseases such as musculoskeletal and urinary tract disorders. ${ }^{18}$ In such a population, the specificity of the clinical indicators would be reduced as well as their power of sparing unnecessary imaging.

Gifford et al have recently reviewed studies on the ability of rules for clinical prediction to lead to the suspicion of reversible causes of dementia and to determine who should undergo CT or MRI. ${ }^{1}$ The authors, as well as others, ${ }^{19}$ found that the most accurate prediction rule was that of Dietch, ${ }^{20}$ requiring imaging in patients with recent onset (less than 1 month) of cognitive symptoms, fluctuations or acute changes (within 48 hours) of cognitive function, focal signs or symptoms, papilloedema or visual field deficits, headache, history of recent head trauma or malignant tumour, seizures, history of stroke, urinary incontinence, or gait ataxia or apraxia. However, even Dietch's rule could not attain $100 \%$ sensitivity ${ }^{1}$ - the benchmark figure that most clinical neurologists and psychiatrists consider acceptable in the detection of reversible causes. ${ }^{16}$ The bottom line is that, although the vast majority of scans carried out in patients with cognitive deterioration are negative, no combination of first line (clinical and laboratory) signs can identify cases of reversible or treatable causes with $100 \%$ sensitivity and reasonably high specificity. ${ }^{17} 2122$

The role of imaging is emphasised also by the recently available antidementia drugs (the cholinesterase inhibitors donpezil, rivastigmine, and galantamine). These have relatively specific effects and indications in the different forms of dementia, being very effective in dementia with Lewy bodies, ${ }^{23}$ moderately effective in Alzheimer's disease, ${ }^{24}$ definitely uneffective in the cognitive symptoms of progressive supranuclear palsy, ${ }^{25}$ and of unknown effectiveness in frontotemporal and vascular dementia. As structural imaging might help in the defferential diagnosis of these diseases (see later), suggesting that the routine use of CT and MRI might be indicated irrespective of their ability to rule out forms of reversible dementia.

Whether CT or MRI should be preferred is an issue that can hardly be solved for the lack of appropriately designed studies. Apart from the obvious practical considerations of cost and accessibility, some features favour CT whereas others favour MRI. On the one hand, CT seems sufficient to rule out reversible causes of dementia ${ }^{19}$ and is more specific in the detection of cerebrovascular disease, ${ }^{26}$ whereas MRI might be more helpful in the differential diagnosis of the forms of degenerative dementia ${ }^{27-31}$ and more sensitive to early or preclinical cases of Alzheimer's disease (see later). ${ }^{32}$ However, direct CT-MR comparisons have not been carried out.

Lastly, rescanning with MRI after CT has led to the diagnosis might be indicated in cases of chronic subdural haematoma eligible for surgical treatment. Tsutsumi et al have shown that the presence of a non-high intensity lesion on T1 weighted MR is a predictor of rebleeding independently of the surgical technique. ${ }^{33}$

\section{Take home messages}

- No combination of clinical and laboratory signs can identify cases of reversible or treatable causes with $100 \%$ sensitivity

- CT without contrast is necessary (and sufficient) to rule out surgical causes of dementia

\section{Detecting cerebrovascular disease}

LARGE AND SMALL VESSEL CEREBROVASCULAR DISEASE

Detecting cerebrovascular disease is relevant in that cerebrovascular lesions occur in more than $30 \%$ of patients with Alzheimer's disease ${ }^{34}$ and might be responsible for a large share of all the cases of cognitive impairment at the population level. ${ }^{65}$ Cerebrovascular disease can be due to large or small vessel disease, giving different clinical and imaging pictures. The most frequent vascular cause of cognitive deterioration is subcortical damage (lacunes and diffuse white matter lesions) due to lesions of the microvessels due to hypertension and diabetes. ${ }^{34}{ }^{36}$ Both CT and MR are known to be sensitive to cerebrovascular disease as with these techniques it has been possible to detect occult (with no signs or symptoms) cerebrovascular disease in mildly impaired as well as cognitively unimpaired patients in some epidemiological and clinical studies. ${ }^{37-39}$ Brain MRI is more sensitive than CT, particularly to white matter lesions. ${ }^{40}$

Diagnosing multi-infarct dementia-in patients with large vessel disease - is usually straightforward for the high frequency of a history of multiple strokes and the presence of obvious focal signs on neurological examination. In these cases, CT or MRI are usually confirmatory and do not add much information to history and clinical findings. Subcortical vascular disease is different because clinical findings are more uncertain. This disease is characterised by a variable combination of cognitive impairment with mainly frontal and dysexecutive features and mild memory disturbances, depression, gait disturbances with mild parkinsonian features, incontinence, falls, and minor neurological signs such as mildly increased and asymmetric deep tendon reflexes and mild Babinski's sign. ${ }^{41}$ The early stages of the disorder are not disabling enough to be categorised as dementia ${ }^{6}$ and are sufficiently mild to significantly overlap with highly prevalent diseases and conditions of older age of a non-cerebrovascular origin such that the clinical picture is often poorly specific. The demonstration of subcortical cerebrovascular disease by CT or MRI can be the key diagnostic information.

In some cases, subcortical cerebrovascular disease gives predominantly depressive or parkinsonian symptoms-socalled "vascular depression" and "vascular parkinsonism". ${ }^{42-44}$ However, to what extent the syndromes of subcortical vascular cognitive impairment, vascular depression, and vascular parkinsonism overlap with each other is unclear as well as the presence of imaging correlates specific to each condition.

THE VASCULAR BURDEN IN ALZHEIMER'S DISEASE

Vascular changes are often present in Alzheimer's disease and seem to contribute to determine cognitive impairment in an additive fashion. ${ }^{4-47}$ Because it has been suggested that the response of patients with Alzheimer's disease to acetylcholinesterase inhibitors might differ according to the magnitude of the cerebovascular contribution, ${ }^{48}$ estimating the vascular burden in such patients might also help understand part of the variability of the response to drug treatment of Alzheimer's disease.

For isolated subcortical cerebrovascular disease, its association with Alzheimer's disease is not characterised by 
a particular clinical picture, and the potential added diagnostic value of imaging is again huge. As the typical changes of Alzheimer's disease-regional atrophy in the medial temporal lobe-are not specific, being present also in vascular dementia, ${ }^{7} 271$ the judgement of causality in a given patient should ideally involve firstly the estimate of the contribution of the vascular changes to cognitive impairment ("is the whole clinical picture attributable to vascular changes?") and, if the answer is negative, that of atrophy net of the effect of vascular changes ("if not, how large is the contribution of degenerative disease?"). ${ }^{49}$ Although such quantitative estimates have been attempted on postmortem material where ischaemic changes and Alzheimer's disease specific lesions can be directly measured, ${ }^{45-47}$ attempts in the living patient have given only qualitative indications of the presence or absence of degeneration with vascular changes, ${ }^{50} 51$ thus failing to capture the range of the different degrees of the mixture of vascular and degenerative lesions.

\section{CRITICAL ISSUES TO MEASURE CEREBROVASCULAR DISEASE} WITH CT AND MRI

Some critical gaps in knowledge prevent a satisfactory answer being given to the issue of the contribution of subcortical cerebrovascular disease to cognitive impairment alone or in addition to Alzheimer's disease. Notably, the relations of number, size, site, and type of lesions with cognitive impairment are unclear. Although pathological studies have shown a relation of number of ischaemic lesions, total volume of ischaemic tissue, and left side and bilateral involvement with cognitive performance, the amount of explained variance is low. ${ }^{40}$ In analogy with the case of multiple sclerosis, ${ }^{52}$ this might be due to normally appearing white matter bearing tiny but widespread lesions.

A recent finding supporting this hypothesis is that of Yamauchi et al who have shown that corpus callosum atrophy - a proxy of global axonal loss - is strongly associated with cognitive impairment independently of the effect of white matter lesions that can be appreciated on T2 weighted MR images..$^{53}$ Moreover, lacunes and other white matter changes might have different powers to cause cognitive impairment, as the first consist of complete interruption of axonal fibres on pathology, whereas the second incomplete infarction and demyelination. ${ }^{3654}$ However, in a necropsy study of demented patients without Alzheimer changes, Esiri unexpectedly found that dementia was associated with the presence of microvascular deep white matter changes but not with macroscopic infarctions. ${ }^{55}$ Similar pathological findings had been reported 10 years earlier by del Ser et al, ${ }^{56}$ and more recent in vivo MRI based data also support this view. ${ }^{7}$

Lastly, not all white matter changes detected by imaging techniques are alike. Patchy areas of attenuation in the white matter on CT and irregular and confluent white matter hyperintensities on MRI have been suggested to be due to ischaemia, whereas other types of hyperintensities seem to be more often related to non-ischaemic changes. ${ }^{54}{ }^{57}$ Punctate lesions can be an early stage of confluent lesions, but sometimes there seems to be no pathological substrate. ${ }^{54}$ Diffuse and homogeneous periventricular hypodensity of the white matter on CT and smooth periventricular hyperintensities on MRI are due to increased water content, demyelination, and subependimal gliosis caused by non-ischaemic alterations of the periventricular fluid dynamics. ${ }^{54} 58$

THE TOOLS: RATING SCALES ON CT AND MR

Some visual rating scales to quantify cerebrovascular changes in the white matter have been developed, based on both CT and MR. ${ }^{59}$ Most record number, size, and site of

\section{Take home messages}

- CT or MRI are necessary to recognise cognitive impairment due to subcortical vascular disease

- As well as infarcts and lacunes, patchy lesions on CT and irregular and confluent white matter hyperintensities on MRI are indicative of cerebrovascular disease whereas smooth periventricular hyperintensities are not

- Visual rating scales should be used to quantify the cerebrovascular burden in patients with cognitive impairment

the lesions, but none gives different weights to the different types of lesions and none incorporates information on qualitative features. A review on rating scales of changes in white matter has led a European Task Force to conclude that "the ideal rating scale does not yet exist". ${ }^{59}$ Although this problem is certainly not unique to the assessment of the cerebrovascular burden in dementia, research groups world wide are trying to devise rating scales based on CT or MRI that can be both accurate and closely related to cognitive performance. ${ }^{364060}$ The inclusion of qualitative features of changes in white matter (for example, homogeneous periventricular versus patchy rarefaction on CT or punctate versus confluent hyperintensities on MRI) as well as weighting differently different types of lesions (for example, lacunes versus non-lacunar lesions) might prove useful.

Lastly, whether CT or MRI should be used to detect cerebrovascular disease is unclear. Criteria for subcortical vascular dementia have been recently developed including indications on number, size, site, and type of lesions on both CT and MRI. ${ }^{46}$ because of the greater specificity of CT, some authors have suggested that this should be preferred to MRI to detect subcortical vascular changes associated with dementia. ${ }^{26}$ However, the earliest stages of subcortical vascular dementia (called vascular cognitive impairment-no dementia) ${ }^{6}$ might need greater sensitivity and MRI might be more accurate. Future work will need to compare the validity of CT and MRI based criteria versus clinical and pathological findings in cognitive impairment with and without dementia due to subcortical cerebrovascular disease.

Until the ideal rating scale for subcortical cerebrovascular disease is devised, it is useful if clinicians choose one of the available rating scales and use it routinely in each patient with cognitive impairment. This will allow us to build a common clinical unity that will make adoption of a common instrument easier when it becomes available. Scheltens et $a \bar{l}^{9}$ have supported the use of the scales of Tarvonen-Schröder et $a l^{61}$ and Rezek et $a l^{62}$ to rate cerebrovascular disease on CT and those of Scheltens et $a l^{73}$ and Fazekas et al on MRI. ${ }^{64} 65$

\section{Detecting brain atrophy}

DIFFERENTIATING ALZHEIMER'S DISEASE AND MILD COGNITIVE IMPAIRMENT FROM NORMAL WITH CT AND MRI

Brain atrophy is present in Alzheimer's disease and other degenerative dementias. Indeed, the NINCDS-ADRDA criteria indicate that the diagnosis of probable Alzheimer's disease is supported by evidence of brain atrophy on CT. ${ }^{66}$ However, subjective visual assessment of global atrophy - at least when carried out in an unstandardised fashion - is poorly reliable for the very low intrarater and interrater agreement, ${ }^{14}{ }^{15}$ such that it usually does not add significant diagnostic information. Quantitative methods to rate atrophy, based on standardised protocols and 
focused in the medial temporal lobe region-known to be involved early in Alzheimer's disease-have been developed based on CT and MRI (table 1).

A review by DeCarli et al dating back to $1990^{67}$ about the ability of CT to help in the discrimination of patients with Alzheimer's disease from non-demented elderly persons showed that, on average, sensitivity and specificity figures of simple (subjective visual or linear measures) ratings of global atrophy were rather low $(32 \%$ to $68 \%$ and $81 \%$ to $90 \%$, respectively). When more complex methods such two dimensional or three dimensional measurements were made, the accuracy increased (63\% to $88 \%$ sensitivity and $90 \%$ specificity). It should be noted that these figures are not higher than those reported for the accuracy of the clinical versus the pathological diagnosis of Alzheimer's disease ${ }^{68-70}$ arguing against a high added diagnostic value, at least in research settings.

Starting from the early 1990 s, researchers' attention has focused on MRI due to its greater flexibility and image accuracy, and studies on CT have become progressively rarer. Few studies have been published in the past decade on the usefulness of CT in the diagnosis of dementia and Alzheimer's disease. Nevertheless, the technological advances on CT machines (greater number of detectors, larger field of view, thinner slices, more detailed matrix, and lower scan time and less movement artifacts) have improved image quality and tissue/CSF contrast. This leads to greater accuracy with simple indicators of atrophy directed to measure changes in the medial temporal lobe, where the pathological lesions of Alzheimer's disease first develop. Indeed, most studies have used linear measures of mesial temporal lobe thickness, temporal horn width, and hippocampal fissure width often taken directly on the CT film. ${ }^{71-76}$ Image acquisition was usually on a specifically angulated plane $\left(-20^{\circ}\right.$ on the orbitomeatal line) that allows the better appreciation of the medial temporal lobe structure. The sensitivity of the measures to separate patients with Alzheimer's disease from non-demented elderly controls ranged in the different studies between $70 \%$ and $92 \%$, and specificity between $80 \%$ and $95 \%$. Computing average sensitivity and specificity figures by weighting for the number of subjects included in each study gives figures of $84 \%$ and $92 \%$, respectively. It should be noted that most studies were on patients with Alzheimer's disease of moderate severity. More complex analytical tools (volumetrics of the lateral and third ventricle) did not contribute to greatly enhance accuracy (sensitivity $70 \%$ to $74 \%$ and specificity $83 \%$ to $95 \%){ }^{77} 78$

Brain MRI has advantages over CT, among which are the lack of bone hardening artifacts-particularly disturbing in the medial temporal lobe regions - the possibility of obtaining oblique and transverse cuts, and discriminating the white from the grey matter of the medial temporal lobe structures. Brain MRI has allowed us to get closer to the pathological basis of Alzheimer's disease by directly measuring tissue loss in the medial temporal lobe regions (hippocampus, amygdala, and enorhinal cortex). Protocols have been developed, most requiring that a trained person familiar with the minute anatomy of the nervous system traces the boundaries of the structure of interest on a computer screen with a pointing device (mouse, trackball, or optical pen) on each of the slices where the structure can be appreciated. Before measurement, it is sometimes necessary to transfer the digital image to a workstation external to the MRI machine and use specifically designed tracing software. This approach has shown an enormous research potential, allowing the definition of the the factors affecting brain structure in health and disease. ${ }^{79-81}$

The studies addressing the discriminative power of MRI to separate patients with Alzheimer's disease from non-demented elderly people have recently been reviewed by Bosscher and Scheltens. ${ }^{82}$ The authors found 13 studies published from 1992 to 1999 measuring hippocampal and medial temporal structures volumes and totalling 425 patients with Alzheimer's disease and 409 non-demented controls. Sensitivity ranged between $80 \%$ and $94 \%$ (weighted average $85 \%$ ) and specificity between $60 \%$ and $100 \%$ (weighted average $88 \%$ ). They also reviewed 10 studies that used standardised visual ratings or linear measures published between the years 1992 and 2000 and totalling 450 patients with Alzheimer's disease and 515 controls. The accuracy figures were similar to those of the more complex analysis, sensitivity ranging between $41 \%$ and $100 \%$ (weighted average $86 \%$ ) and specificity between $66 \%$ and $96 \%$ (weighted average $85 \%$ ). The disease severity of patients with Alzheimer's disease in the MRI studies was generally in the mild to moderate range. It is interesting to note that the accuracy figures of the above reviewed CT studies-employing less complex techniques on relatively more advanced patients - were of similar magnitude $(84 \%$ and $92 \%)$. Thus, in experimental settings, both simple and complex tools based on CT and MRI have been able to separate patients with Alzheimer's disease from non-demented controls, with reasonably high accuracy.

Hippocampal volumetrics with MRI has been tested to diagnose very early Alzheimer's disease, when symptoms are limited to memory and disability has not yet developed (mild cognitive impairment). ${ }^{5}$ Petersen et al have measured hippocampal volumes with MRI in 80 patients with mild cognitive impairment and found that, although the values were in the low end of the age, sex, and head size specific distribution of cognitively unimpaired controls, the overlapping was substantial (sensitivity of $37 \%$ for specificity of $95 \%) .^{32}$ Although greater atrophy was associated with greater risk of conversion to Alzheimer's disease, only the greatest degrees of atrophy were sufficiently predictive, all of the 13 patients with hippocampal volumes $2.5 \mathrm{SD}$ or more below average developing Alzheimer's disease within 4 years versus only one of the 13 with hippocampal volumes above average. The usefulness of CT in the recognition of those with mild cognitive impairment who will convert to Alzheimer's disease has not yet been tested.

It should be underlined that predicting and recognising mild cognitive impairment is an area where imaging techniques might yield the greatest added value. In fact, half of those with mild cognitive impairment do not convert to Alzheimer's disease within the reasonably long period of time of 5 years, consistent with the possibility that present

Table 1 Measurements of regional brain atrophy by different protocols

\begin{tabular}{|c|c|c|c|c|c|}
\hline & $\begin{array}{l}\text { Acquisition: specific } \\
\text { plane and slice thickness }\end{array}$ & $\begin{array}{l}\text { Preprocessing } \\
\text { of image }\end{array}$ & $\begin{array}{l}\text { Can be taken from } \\
\text { radiological films }\end{array}$ & $\begin{array}{l}\text { Specific hardware } \\
\text { /software }\end{array}$ & $\begin{array}{l}\text { Expertise of } \\
\text { rater/tracer }\end{array}$ \\
\hline \multicolumn{6}{|l|}{ CT: } \\
\hline Linear measures & Yes & No & Yes & No & Average \\
\hline \multicolumn{6}{|l|}{ MRI: } \\
\hline Visual ratings & Yes & No & Yes & No & High \\
\hline Linear measures & Yes & No & Yes & No & Average \\
\hline Surface/volume measures & Yes & Yes & No & Yes & High \\
\hline
\end{tabular}


clinical criteria to recognise the condition might be poorly specific. Atrophy markers might help increasing specificity. A promising technique for this has been developed by Fox et al, requiring repeated MR scans over time and dedicated postprocessing of the digital images. The technique is sensitive in detecting brain tissue losses as small as $0.5 \%$ of the total brain volume. ${ }^{83}$ Recent improvements in this technique allow the detection of regional instead of global atrophy. ${ }^{84}$

\section{Differentiating Alzheimer's disease from other forms of degenerative dementia}

Some infrequent types of dementia have a relatively specific imaging pattern and, with the contribution of the clinical findings, can be easily distinguished from Alzheimer's disease. The atrophy pattern perhaps most specific is that of extremely severe frontopolar and temporopolar atrophy of the cortical layer and white matter associated with often asymmetric (left greater than right) dilatation of the frontal (ballooning) and temporal horns. ${ }^{85}$ These patients usually have a cognitive behavioural syndrome consistent with frontotemporal dementia or progressive aphasia ${ }^{85} 86$ and on postmortem examination show Pick's changes. ${ }^{85}$ However, some rare patients with Pick's disease show severe but more posterior atrophy in the parietal lobes with a syndrome that can suggest corticobasal degeneration. ${ }^{87} 88$ Posterior cortical atrophy involving mainly the occipital lobes can be due to Alzheimer's disease ${ }^{89}{ }^{90}$ or Creutzfeldt-Jakob disease. ${ }^{90}{ }^{91}$ In about $70 \%$ of cases of sporadic Creutzfeldt-Jakob disease, T2 weighted MRI shows marked hyperintensities in the basal ganglia (caudate and putamen) and mild hyperintensities in the thalamus. ${ }^{92} 93$ By contrast, in the new variant Creutzfeldt-Jakob disease hyperintensities in the basal ganglia are mild and those in the thalamus are marked ("pulvinar sign") and constitute a moderately sensitive (80\%) but highly specific (100\%) indicator. ${ }^{94}{ }^{95}$

However, the vast majority of patients with degenerative dementia show changes less striking than those described, and CT and MR findings are, unfortunately, poorly specific. In this area, virtually all research work has been done with MRI and has considered the differentiation of Alzheimer's disease from non-Pick's frontotemporal dementia, Levy body dementia, and parkinsonian dementia. Generally, studies using a single region to differentiate the groups have found remarkable overlap among groups, and the accuracy of the separation was lower than that of Alzheimer's disease with non-demented controls. Some research groups have shown that the hippocampal volume of parkinsonian dementia, Lewy body, and frontotemporal dementia was intermediate between that of Alzheimer's disease of similar severity and non-demented controls. ${ }^{27} 3031$ Sensitivity figures for the detection of the nonAlzheimer's disease dementias were low, ranging-with specificity set at $90 \%$ - between $30 \%$ and $67 \%$. Moreover, to be transferable to the differential diagnostic process of an individual patient in clinical practice, the single region approach should define severity specific atrophy measures, a process requiring large group sizes.

A different approach tries to identify patterns of atrophy, where atrophy in a given region is judged compared with that of another region within the same diagnostic group or person. Studies based on MRI and pathology of extrapyramidal diseases have shown variable atrophic changes in some brain structures ${ }^{96} 97$ and supported the view that the combination of information on atrophy in two or more structure is a more accurate discriminator than atrophy in only one structure. Schulz et al have shown that idiopathic Parkinson's disease, multiple system atrophy, and progressive supranuclear palsy can be differentiated from each

\section{Take home messages}

- In the absence of relevant cerebrovascular disease, CT and MRI appropriately performed and rated allow medial temporal lobe atrophy in Alzheimer's disease to be quantified

- The added diagnostic value of atrophy ratings might be significant in routine clinical settings

- The role of atrophy ratings in mild cognitive impairment is uncertain

other with MRI based volumetric measurements of caudate nucleus, putamen, brainstem, and cerebellum. ${ }^{97}$

Patterns of atrophy different from Alzheimer's disease have been identified in vivo for patients with frontotemporal dementia (table 2). ${ }^{3098}$ Whereas patients with Alzheimer's disease show moderate to severe atrophy of the hippocampal and amygdalar structures and mild atrophy of the frontal lobes, patients with frontotemporal dementia show severe frontal and temporal atrophy and much milder atrophy in the medial temporal lobe structures. In a previous study with linear measures of medial temporal and frontal atrophy, we found that the separation of patients with frontotemporal dementia from patients with Alzheimer's disease in a multivariable discriminant model was relatively low (sensitivity and specificity of $79 \%$ and $61 \%$ ). The pattern approach has recently been applied to Lewy body dementia by British and Japanese research groups, but the contrasting findings do not allow the recognition of a pattern significantly different from that of patients with Alzheimer's disease (table 2).$^{28}{ }^{31}$ However, if applied to the individual patient rather than on a group basis - which still has to be done-the pattern approach should be more independent of disease severity than the single region approach. Highly technological variations are being developed that allow the outlining of patterns of atrophy within a given structure (for example, the hippocampus) and might provide a more accurate marker of Alzheimer's disease to be used in the differential diagnosis from other degenerative dementias and in the estimate of the interrelation between coexistent vascular and degenerative changes. ${ }^{100} 101$

THE TOOLS: BALANCING ACCURACY AND FEASIBILITY

The projected increasing prevalence of Alzheimer's disease that will occur in the next decades ${ }^{102}$ and the increasing therapeutic options will require that diagnostic skills of the highest possible level be spread at the population level. The

Table 2 Qualitative patterns of atrophy in some frequent forms of degenerative dementia

\begin{tabular}{lllll}
\hline & $A D$ & FTD & DLB & PDD \\
\hline Frontal lobes & \pm & +++ & - & \pm \\
Frontal horns of lateral ventricles & \pm & +++ & & \\
Temporal lobes & + & +++ & \pm & \\
Temporal horns of lateral ventricles & +++ & ++++ & & \\
Amygdala & ++ & & ++ & \\
Entorhinal cortex & ++ & + & & \\
Hippocampus & +++ & + & ++ & ++++
\end{tabular}

Based on volumetric studies ${ }^{27-31}$ and personal unpublished data on FTD.

$\mathrm{AD}=$ Alzheimer's disease; $\mathrm{FTD}=$ frontotemporal dementia; $\mathrm{DLB}=$ dementia with Lewy bodies; PDD=Parkinson's disease with dementia.

Marks denote volumetric loss (or ventricular enlargement) between 0 and -1 $\mathrm{SD}$ from the control distribution $( \pm)-1$ and $-1.5(+) ;-1.5$ and $-2(++) ;-2$ and $-2.5(+++) ;<-2.5(++++)$. When this information was not available, \pm denoted a loss between $0 \%$ and $10 \%$ of a control group; + between $10 \%$ and $20 \%$; ++ between $20 \%$ and $30 \%$; +++ between $30 \%$ and $40 \%$; ++++ over $40 \%$. In the case of ventricles, $\%$ enlargement was computed with the formula $(1-1 / \%$ enlargement) to preserve comparability with tissue structures. The -sign denotes no atrophy (or ventricular enlargement). 
works reviewed above show that accurate atrophy measurement tools are available for both CT and MRI, which differ as to practical technical requirements. In routine practice, the need is that of achieving the greatest added diagnostic value in the most feasible way.

Studies explicitly considering the added value of different methods of analysis of structural images are few. Wahlund et al have compared standardised visual ratings of the medial temporal lobe with hippocampal volumetrics on MRI. They found that the first provided additional information in the separation of patients with Alzheimer's disease from controls when considered in conjunction with the mini mental state examination (MMSE) (sensitivity increased from $81 \%$ with the MMSE alone to $93 \%$ and specificity from $95 \%$ to $98 \%$ ) and that the increase of information was greater than that provided by hippocampal volumetrics (from $81 \%$ to $88 \%$ and from $95 \%$ to $96 \%) .{ }^{103}$ These results need to be tested in routine clinical settings by other research groups. Comparisons between even more feasible tools-such as linear measures developed as indirect markers of hippocampal atrophy on $\mathrm{CT}^{104}$ and hippocampal volumetrics on MRI-have not been performed.

It should be highlighted that the added diagnostic values of imaging techniques in routine settings might be higher than indicated by studies carried out in experimental settings. Indeed, the selection of the diagnostic groups in the experimental settings follows an extensive investigation including detailed history taking, assessment of physical health through clinical, laboratory, and instrumental examinationss, and neuropsychological testing. For this reason, when, for example, Alzheimer's disease is suspected before CT or MR, the likelihood that the patient indeed has Alzheimer's disease in such centres is relatively high. This view is also supported by the high accuracy ( $87 \%$ to $100 \%$ ) of the pathologically confirmed clinical diagnosis of probable Alzheimer's disease in some research centres ${ }^{105} 106$ and by the increase of specificity over time in expert centres. ${ }^{107}$ In these centres, CT and MRI can obviously provide little or no additional information, due to a ceiling effect. However, in Europe as well as in the United States most patients with dementia are not diagnosed in expert research centres but in first or second level clinical referral centres, where the amount of time, personnel, and accessory evaluations are lower, and the prior probability is likewise lower. In this setting, the availability of CT or MRI based disease markers might be of greater added value. A further potentially useful application of these markers is in multicentre clinical trials, where they might enhance the homogeneity of the diagnostic accuracy throughout centres.

The issue of where and who should perform the ratings is also a relevant issue. At the present time, the general rule in European countries is that CT and MR images are printed on film, and read by a radiologist who seldom has a specific interest or training in dementia. Table 1 shows that linear measures on CT and visual ratings and linear measures on MRI do not need to be preprocessed and can be taken on conventional films. Either the radiologist or the clinician (neurologist or psychiatrist) can take the measures. On the contrary, surface and volume measures do require postprocessing of images as well as specific hardware and software. Presently, such measures are taken in research centres by neurologists or psychiatrists where images can be swiftly transferred in digital format from the MR scanner of the radiology department to a microcomputer or workstation where the image is postprocessed. Measurements need to be done with specific software (some of which are freely available-see for example, NIH Image available on http://rsb.info.nih.gov/nih-image/ and others listed in http://

\section{Take home messages}

- Patients with sporadic Creutzfeldt-Jakob disease show hyperintensities in the striatum on T2 weighted MRI and those with new variant Creutzfeldt-Jakob disease show hyperintensities in the posterior thalamus (pulvinar sign)

- Patients with Pick's disease show severe and markedly asymmetric anterior frontal and/or temporal atrophy of the cortex and white matter with severe enlargement of the ventricular horns

- Patients with Alzheimer's disease, frontotemporal dementia, Lewy body dementia, and dementia of Parkinson's disease have different patterns of regional atrophy; these are subtle, but can be detected with specific postprocessing of MRI, and their diagnostic yield needs to be elucidated

www.neuroguide.com/neurosoft_1.html\#programs/) and sometimes with specific hardware platforms such as SUN workstations (DeCarli's QUANTA ${ }^{108}$ or Mayo Clinic's ANALYZE at http://www.mayo.edu/bir/). These merely practical issues are none the less a huge barrier to the use of such methods in routine clinical practice. Despite the present limitations, the shift of the management of radiological images from analog to fully digital systems such as the Picture Archive and Communications System (for example, that on http://www.cemaxicon.com/) will in the future make the transfer of digital images from the site of acquisition to peripheral sites much easier and postprocessing more feasible.

\section{Future developments and conclusions}

Some novel imaging techniques are being developed that might in the future significantly alter the clinical practices. The development of tools such as probabilistic atlases based on traditional MR images might help identify subtle structural changes by comparing the scan of a patient with a large database of normal controls on a voxel by voxel basis, thus obtaining probabilistic maps localising where the atrophic changes have taken place (Mazziotta Neuroimage 1995). Alternatively, disease specific atlases might help in the differentiation of the different dementia forms. ${ }^{109}$ Other MRI based techniques such as diffusion weighted imaging, magnetisation transfer, and spectroscopy ${ }^{110}$; imaging tools based on SPET and PET that allow the visualisation of the accumulation of $\beta$-amyloid in vivo $^{111-113}$; and new functional $M R$ imaging protocols ${ }^{114}$ might allow the better estimation of the frequency and severity of Alzheimer's changes alone or associated with cerebrovascular disease, and improve the differential diagnosis of dementia and the prognosis of the conversion of mild cognitive impairment into Alzheimer's disease.

However, while these exciting technological options get sufficiently advanced to be used in routine clinical settings, less technological and perhaps less exciting - but none the less useful-tools are already available that allow us to extract more and more useful information from conventional CT and MR images. Some are sufficiently feasible and accurate to be used in routine clinical settings. Clinicians should choose one tool to rate the cerebrovascular burden and one to rate medial temporal lobe atrophy and use them routinely on patients with memory or other cognitive disturbances or suspected Alzheimer's disease. According to local conditions, CT or MRI based tools might be preferred as well as the level of technical complexity of atrophy measurement tools.

It should be noted that the rapidly changing clinical behaviours of physicians brought about by the availability 
Take home message

- At least linear measures of medial temporal lobe atrophy on CT or visual rating scales of hippocampal formation atrophy on MRI should be used in each patient with cognitive impairment

of new drugs with different indications and different efficacy profiles ${ }^{24}$ will increase the relative value of early diagnosis and of the differential diagnosis among different dementia forms and, consequently, of imaging markers. Cost-effectiveness studies are long and elaborate, and it is likely that when these become available, the clinical framework where they were conceived will be no longer applicable to the new reality. This is, for example, the case of a cost-effectiveness review of studies in the 1980s and early 1990 s on the use of CT as a rule out diagnostic tool. ${ }^{115}$ As the studies on which the review is based were carried out before cholinesterase inhibitors were available and these drugs that have been shown to increase quality adjusted life-years ${ }^{116}$ the review does not allow us to address the fall out of CT on correct diagnosis of Alzheimer's disease, treatment, and quality of life.

Lastly, it should be kept in mind that the reliance on technology does not itself enhance the quality of medical care. ${ }^{117}$ Indeed, one of the most important implications of any technological innovation is an increase in relative value among clinical diagnostic skills of history taking and mental status examination, which will remain largely beyond the reach of technology. ${ }^{118}$ It is from the careful blend of these exquisitely clinical abilities with technologicaldiagnostic and therapeutical - aids that the physician can understand the patient in his personal, social, and medical dimensions and provide optimal treatment.

G B FRISONI

Laboratory of Epidemiology and Neuroimaging, IRCCS San Giovanni di Dio-FBF, via Pilastroni 4, I-25125 Brescia, Italy

Correspondence to: G B Frisoni

papers@centroAlzheimer.it

1 Gifford DR, Holloway RG, Vickrey BG. Systematic review of clinical prediction rules for neuroimaging in the evaluation of dementia. Arch Intern prediction rules for neuro

2 Mazziotta JC, Toga AW, Evans A, et al. A probabilistic atlas of the human brain: theory and rationale for its development. The International Consortium for Brain Mapping (ICBM). Neuroimage 1995;2:89-101.

3 Perry S, Thamer M. Medical innovation and the critical role of health technology assessment. $\mathcal{F} A M A$ 1999;282:1869-72.

4 Maynard A. Evidence-based medicine: an incomplete method for informing treatment choices. Lancet 1997;349:126-8.

5 Petersen RC, Smith GE, Waring SE, et al. Mild cognitive impairment. Clinical characterization and outcome. Arch Neurol 1999;56:303-8.

6 Rockwood K, Wentzel C, Hachinski V, et al. Prevalence and outcomes of vascular cognitive impairment. Vascular Cognitive Impairment Investigators of the Canadian Study of Health and Aging. Neurology 2000;54:44751 .

7 Fein G, Di Sclafani V, Tanabe J, et al. Hippocampal and cortical atrophy predict dementia in subcortical ischemic vascular disease. Neurology 2000; 55:1626-35.

8 The Canadian Study of Health and Aging Working Group. The incidence of dementia in Canada. Neurology 2000;55:66-73.

9 Graham JE, Rockwood K, Beattie BL, et al. Prevalence and severity of cognitive impairment with and without dementia in an elderly population. nitive impairment with and

10 Banta D, Oortwijn W. Health technology assessment and health care in the European Union. Int $\mathcal{F}$ Technol Assess Health Care 2000;16:626-35.

11 Rossor MN. Management of neurological disorders: dementia. $f$ Neurol Neurosurg Psychiatry 1994;57:1451-6.

12 Decreto del ministero della Sanità 28 maggio 1999, n 329 (in supplemento ordinario 174/L alla "Gazzetta Ufficiale" 226 del 25 settembre 1999), concernente: Regolamento recante norme di individuazione delle malattie croniche e invalidanti ai sensi dell'articolo 5, comma 1, lettera a), del decreto legislativo 29 aprile 1998:124. Roma: Consiglio die Ministri.

13 Schneider R, Kluge R, Willmes K. Interrater agreement for CT scans of patients with lacunar infarcts and leuko-araiosis. Acta Neurol Scand 1991;84:527-30.

14 Scheltens P, Pasquier F, Weerts JG, et al. Qualitative assessment of cerebral atrophy on MRI: inter- and intra-observer reproducibility in dementia and normal aging. Eur Neurol 1997;37:95-9.

15 Leonardi M, Ferro S, Agati R, et al. Interobserver variability in CT assessment of brain atrophy. Neuroradiology 1994;36:17-9.

16 Haydel MJ, Preston CA, Mills TJ, et al. Indications for computed tomography in patients with minor head injury. N Engl F Med 2000;343:100-5.
17 Chui H, Zhang Q. Evaluation of dementia: a systematic study of the usefulness of the American Academy of Neurology's practice parameters. Neurology 1997;49:925-35.

18 Kokmen E, Ozsarfati Y, Beard CM, et al. Impact of referral bias on clinical and epidemiological studies of Alzheimer's disease. F Clin Epidemiol 1996; 49:79-83.

19 Alexander EM, Wagner EH, Buchner DM, et al. Do surgical brain lesions present as isolated dementia? A population-based study. 7 Am Geriatr Soc 1995;43:138-43.

20 Dietch JT. Computerized tomographic scanning in cases of dementia. West $\mathcal{F}$ Med 1983;138:835-7.

21 Branton T. Use of computerized tomography by old age psychiatrists: an examination of criteria for investigation of cognitive impairment. Int $\mathcal{F}$ Geriatr Psychiatry 1999;14:567-7

22 Moles JK, Franchina JJ, Sforza PP. Increasing the clinical yield of computerized tomography for psychiatric patients. Gen Hosp Psychiatry 1998;20 282-91.

23 McKeith I, Del Ser T, Spano P, et al. Efficacy of rivastigmine in dementia with Lewy bodies: a randomised, double-blind, placebo-controlled international study. Lancet 2000;356:2031-6.

24 Mayeux R, Sano M. Treatment of Alzheimer's disease. N Engl f Med 1999; 341:1670-9.

25 Litvan I, Phipps MS, Pharr V, et al. A controlled, crossover, randomized, double-blind trial of an anticholinesterase agent (donepezil) in patients with progressive supranuclear palsy [abstract]. Neurology 2000;52 (suppl 3):A192.

26 Lopez OL, Becker JT, Jungreis CA, et al. Computed tomography-but not magnetic resonance imaging-identified periventricular white-matter lesions predict symptomatic cerebrovascular disease in probable Alzheimer's disease. Arch Neurol 1995;52:659-64.

27 Laakso MP, Partanen K, Riekkinen P, et al. Hippocampal volumes in Alzheimer's disease, Parkinson's disease with and without dementia, and in vascular dementia: an MRI study. Neurology 1996;46:678-81.

28 Hashimoto $\mathrm{M}$, Kitagaki $\mathrm{H}$, Imamura $\mathrm{T}$, et al. Medial temporal and whole-brain atrophy in dementia with Lewy bodies: a volumetric MRI study. Neurology 1998;51:357-62.

29 Juottonen K, Laakso MP, Insausti R, et al. Volumes of the entorhinal and perirhinal cortices in Alzheimer's disease. Neurobiol Aging 1998;19:15-22.

30 Frisoni GB, Laakso MP, Beltramello A, et al. Hippocampal and entorhinal cortex atrophy in frontotemporal dementia and Alzheimer's disease. Neurology 1999;52:91-100.

31 Barber R, Ballard C, McKeith IG, et al. MRI volumetric study of dementia with Lewy bodies: a comparison with $\mathrm{AD}$ and vascular dementia. Neurology 2000;54:1304-9.

32 Petersen RC, Jack CR Jr, Xu YC, et al. Memory and MRI-based hippocampal volumes in aging and AD. Neurology 2000;54:581-7.

33 Tsutsumi K, Maeda K, Iijima A, et al. The relationship of preoperative magnetic resonance imaging findings and closed system drainage in the recurrence of chronic subdural hematoma. 7 Neurosurg 1997;87:870-5.

34 Kalaria RN, Ballard C. Overlap between pathology of Alzheimer disease and vascular dementia. Alzheimer Dis Assoc Disord 1999;13(suppl 3):115-23.

35 Forette F, Seux ML, Staessen JA, et al. Prevention of dementia in randomised double-blind placebo-controlled systolic hypertension in Europe (Syst-Eur) trial. Lancet 1998;352:1347-51.

36 Pantoni L, Leys D, Fazekas F, et al. Role of white matter lesions in cognitive impairment of vascular origin. Alzheimer Dis Assoc Disord 1999;13(suppl 3):49-54.

37 Lindgren A, Roijer A, Rudling O, et al. Cerebral lesions on magnetic resonance imaging, heart disease, and vascular risk factors in subjects without stroke. A population-based study. Stroke 1994;25:929-34.

38 Awad IA, Spetzler RF, Hodak JA, et al. Incidental subcortical lesions identified on magnetic resonance imaging in the elderly. I. Correlation with age and cerebrovascular risk factors. Stroke 1986;17:1084-9.

39 DeCarli C, Miller B, Swan GE, et al. Cerebrovascular and brain morphological correlates of mild cognitive impairment in the NHLBI twin study. Arch Neurol (in press).

40 Erkinjuntti T, Bowler JV, DeCarli CS, et al. Imaging of static brain lesions in vascular dementia: implications for clinical trials. Alzheimer Dis Assoc Disord 1999;13(suppl 3):81-90.

41 Erkinjuntti T, Inzitari D, Pantoni L, et al. Research criteria for subcortical vascular dementia in clinical trials. F Neural Transm 2000;59:23-30.

42 Zijlmans JC, Thijssen HO, Vogels OJ, et al. MRI in patients with suspected vascular parkinsonism. Neurology 1995;45:2183-8.

43 O'Brien J, Ames D, Chiu E, et al. Severe deep white matter lesions and outcome in elderly patients with major depressive disorder: follow up study. BMF 1998;317:982-4

44 Guo X, Skoog I, Matousek M, et al. A population-based study on motor performance and white matter lesions in older women. $\mathcal{A} \mathrm{Am}$ Geriatr Soc 2000;48:967-70.

45 Goulding JM, Signorini DF, Chatterjee S, et al. Inverse relation between Braak stage and cerebrovascular pathology in Alzheimer predominant dementia. I Neurol Neurosurg Psychiatry 1999;67:654-7.

46 Esiri MM, Nagy Z, Smith MZ, et al. Cerebrovascular disease and threshold for dementia in the early stages of Alzheimer's disease. Lancet 1999;354: 919-20.

47 Snowdon DA, Greiner LH, Mortimer JA, et al. Brain infarction and the clinical expression of Alzheimer disease. The Nun study. $7 A M A$ 1997;277: 813-7.

48 Kumar V, Anand R, Messina J, et al. An efficacy and safety analysis of Exelon in Alzheimer's disease patients with concurrent vascular risk factors. Eur $\mathcal{f}$ Neurol 2000;7:159-69.

49 Hachinski V, Munoz DG. Cerebrovascular pathology in Alzheimer's disease: cause, effect or epiphenomenon? Ann N Y Acad Sci 1997;826:1-6.

50 Henon H, Pasquier F, Durieu I, et al. Medial temporal lobe atrophy in stroke patients: relation to pre-existing dementia. $\mathcal{F}$ Neurol Neurosurg Psychiatry 1998;65:641-7.

51 Henon H, Pasquier F, Durieu I, et al. Preexisting dementia in stroke patients. Baseline frequency, associated factors, and outcome. Stroke 1997;28:242936.

52 Filippi M, Rocca MA, Martino G, et al. Magnetization transfer changes in the normal appearing white matter precede the appearance of enhancin lesions in patients with multiple sclerosis. Ann Neurol 1998;43:809-14. 
53 Yamauchi $\mathrm{H}$, Fukuyama $\mathrm{H}$, Shio $\mathrm{H}$. Corpus callosum atrophy in patients with leukoaraiosis may indicate global cognitive impairment. Stroke with leukoaraiosis

54 Fazekas F, Kleinert R, Offenbacher H, et al. Pathologic correlates of incidental MRI white matter signal hyperintensities. Neurology 1993;43: 1683-9.

55 Esiri MM. Which vascular lesions are of importance in vascular dementia? Ann N Y Acad Sci 2000;903:239-43.

56 del Ser T, Bermejo F, Portera A, et al. Vascular dementia. A clinicopathological study. 7 Neurol Sci 1990;96:1-17.

57 Goto K, Ishii N, Fukasawa H. Diffuse white-matter disease in the geriatric population. A clinical, neuropathological, and CT study. Radiology 1981;141:687-95.

58 Munoz DG, Hastak SM, Harper B, et al. Pathologic correlates of increased signals of the centrum ovale on magnetic resonance imaging. Arch Neurol 1993;50:492-7.

59 Scheltens P, Erkinjunti T, Leys D, et al. White matter changes on CT and MRI: an overview of visual rating scales. European Task Force on Age-Related White Matter Changes. Eur Neurol 1998;39:80-9.

60 Bowler JV, Steenhuis R, Hachinski V. Conceptual background to vascular cognitive impairment. Alzheimer Dis Assoc Disord 1999;13(suppl 3):30-7.

61 Tarvonen-Schroder S, Kurki T, Raiha I, et al. Leukoaraiosis and cause of death: a 5 year follow up. F Neurol Neurosurg Psychiatry 1995;58:586-9.

62 Rezek DL Morris JC, Fulling KH, et al. Periventricular white matter lucencies in senile dementia of the Alzheimer type and in normal aging. Neurology 1987;37:1365-8

63 Scheltens P, Barkhof F, Leys D, et al. A semiquantative rating scale for the assessment of signal hyperintensities on magnetic resonance imaging. $\mathcal{F}$ Neurol Sci 1993;114:7-12

64 Fazekas F, Chawluk JB, Alavi A, et al. MR signal abnormalities at $1.5 \mathrm{~T}$ in Alzheimer's dementia and normal aging. AfR Am f Roentgenol 1987;149: 351-6.

65 Coffey CE, Figiel GS, Djang WT, et al. Subcortical hyperintensity on magnetic resonance imaging: a comparison of normal and depressed elderly subjects. Am F Psychiatry 1990;147:187-9.

66 McKhann G, Drachman D, Folstein M, et al. Clinical diagnosis of Alzheimer's disease: report of the NINCDS-ADRDA Work Group under the auspices of Department of Health and Human Services Task Force on the auspices of Department of Health and Hum

67 DeCarli C, Kaye JA, Horwitz B, et al. Critical analysis of the use of computer-assisted transverse axial tomography to study human brain in aging and dementia of the Alzheimer type. Neurology 1990;40:872-83.

68 Holmes C, Cairns N, Lantos P, et al. Validity of current clinical criteria for Alzheimer's disease, vascular dementia and dementia with Lewy bodies. $\mathrm{Br}$ F Psychiatry 1999;174:45-50.

69 Blacker D, Albert MS, Bassett SS, et al. Reliability and validity of NINCDSADRDA criteria for Alzheimer's disease. The National Institute of Menta Health Genetics Initiative. Arch Neurol 1994;51:1198-204.

70 Kukull WA, Larson EB, Reifler BV, et al. The validity of three clinical diagnostic criteria for Alzheimer's disease. Neurology 1990;40:1364-9.

71 Jobst KA, Smith AD, Szatmari M, et al. Detection in life of confirmed Alzheimer's disease using a simple measurement of medial temporal lobe atrophy by computed tomography. Lancet 1992;340:1179-83.

72 Pasquier F, Hamon M, Lebert F, et al. Medial temporal lobe atrophy in memory disorders. F Neurol 1997;244:175-81.

73 Soininen H, Reinikainen HJ, Puranen M, et al. Wide third ventricle correlates with low choline acetyltransferase activity of the neocortex in correlates with low choline acetyltransferase activity of the
Alzheimer patients. Alzheimer Dis Assoc Disord 1993;7:39-47.

74 Spanò A, Förstl H, Almeida OP, et al. Neuroimaging and the differential diagnosis of early dementia: quantitative CT scan analysis in patients diagnosis of early dementia: quantitative CT scan analysis in

75 Denihan A, Wilson G, Cunningham C, et al. CT measurement of medial temporal lobe atrophy in Alzheimer's disease, vascular dementia, depression and paraphrenia. Int f Geriatr Psychiatry 2000;15:306-12

76 Kido DK, Caine ED, LeMay M, et al. Temporal lobe atrophy in patients with Alzheimer disease: a CT study. AfNR Am f Neuroradiol 1989;10:5515.

77 DeCarli C, Haxby JV, Gillette JA, et al. Longitudinal changes in lateral venricular volume in patients with dementia of the Alzheimer type. Neurology 1992;42:2029-36.

78 Forstl $\mathrm{H}$, Zerfass $\mathrm{R}$, Geiger-Kabisch C, et al. Brain atrophy in normal ageing and Alzheimer's disease. Volumetric discrimination and clinical correlations. Br f Psychiatry 1995;167:739-46.

79 Gur RC, Turetsky BI, Matsui M, et al. Sex differences in brain gray and white matter in healthy young adults: correlations with cognitive performance. F Neurosci 1999; 19:4065-72.

80 Geroldi C, Laakso MP, DeCarli C, et al. Apolipoprotein E genotype and hippocampal asymmetry in Alzheimer's disease. A volumetric MRI study. $\mathcal{F}$ Neurol Neurosurg Psychiatry 2000;68:93-6.

81 Geroldi C, Pihlajamäki M, Laakso MP, et al. ApoE $\varepsilon 4$ is associated with less frontal and more medial temporal lobe atrophy in AD. Neurology 1999:53: $1825-32$.

82 Bosscher L, Scheltens Ph. MRI of the temporal lobe. In: Qizilbash N, Schneider L, Chui H, et al, eds. Evidence based dementia. Oxford: Blackwell, 2001 (in press)

83 Fox NC, Freeborough PA. Brain atrophy progression measured from registered serial MRI: validation and application to Alzheimer's disease. 7 Magn Reson Imaging 1997;7:1069-75.

84 Scahill RI, Crum WR, Fox NC. Novel methodology for assessment of progression of hippocampal atrophy from volumetric MR scans. Proceedings of the 1st meeting of the Alzheimer's Imaging Consortium. Washington, DC: American Geophysical Union, 8 July 2000
85 Knopman DS, Christensen KJ, Schut LJ, et al. The spectrum of imaging and neuropsychological findings in Pick's disease. Neurology 1989;39:362-8. 86 Mendez MF, Selwood A, Mastri AR, et al. Pick's disease versus Alzheimer's disease: a comparison of clinical characteristics. Neurology 1993;43:289-92. 87 Lang AE, Bergeron C, Pollanen MS, et al. Parietal Pick's disease mimicking cortical-basal ganglionic degeneration. Neurology 1994;44:1436-40.

88 Cambier J, Masson M, Dairou R, et al. Etude anatomo-clinique d'une forme parietale de maladie de Pick. Rev Neurol 1981;137:33-8.

89 Rogelet P, Delafosse A, Destee A. Posterior cortical atrophy: unusual feature of Alzheimer's disease. Neurocase 1996;2:495-501.

90 Victoroff J, Ross GW, Benson DF, et al. Posterior cortical atrophy. Neuropathologic correlations. Arch Neurol 1994;51:269-74.

91 Renner J, Morris J, Storandt M, et al. Progressive posterior cortical dysfunction predicts global dementia [abstract]. Neurology 1992;42(suppl 3):A132.

92 Schroter A, Zerr I, Henkel K, et al. Magnetic resonance imaging in the clinical diagnosis of Creutzfeldt-Jakob disease. Arch Neurol 2000;57:1751-7.

93 Zerr I, Schulz-Schaeffer WJ, Giese A, et al. Current clinical diagnosis in Creutzfeldt-Jakob disease: identification of uncommon variants. Ann Neurol 2000;48:323-9.

94 Zeidler M, Sellar RJ, Collie DA, et al. The pulvinar sign on magnetic resonance imaging in variant Creutzfeldt-Jakob disease. Lancet 2000;355:14128.

95 Will RG, Zeidler M, Stewart GE, et al. Diagnosis of new variant Creutzfeldt-Jakob disease. Ann Neurol 2000;47:575-82.

96 Cordato NJ, Halliday GM, Harding AJ, et al. Regional brain atrophy in progressive supran

97 Schulz JB, Skalej M, Wedekind D, et al. Magnetic resonance imaging-based volumetry differentiates idiopathic Parkinson's syndrome from multiple system atrophy and progressive supranuclear palsy. Ann Neurol 1999;45: s5-74.

98 Frisoni GB, Beltramello A, Geroldi C, et al. Brain atrophy in frontotemporal dementia. $\mathcal{F}$ Neurol Neurosurg Psychiatry 1996;61:157-65.

99 Galton CJ, Gomez-Anson B, Antoun N, et al. Temporal lobe rating scale: application to Alzheimer's disease and frontotemporal dementia $\mathcal{F}$ Neurol Neurosurg Psychiatry 2001;70:165-73.

100 Csernansky JG, Wang L, Joshi S, et al. Early DAT is distinguished from aging by high-dimensional mapping of the hippocampus. Neurology 2000;55:1636-43.

101 Laakso MP, Frisoni GB, Kononen M, et al. Hippocampus and entorhinal cortex in frontotemporal dementia and Alzheimer's disease: a morphometric MRI study. Biol Psychiatry 2000;47:1056-63.

102 Brookmeyer R, Gray S, Kawas C. Projections of Alzheimer's disease in the United States and the public health impact of delaying disease onset. $A m \mathcal{F}$ Public Health 1998;88:1337-42.

103 Wahlund LO, Julin P, Sven-Erik J, et al. Visual rating and volumetry of the medial temporal lobe on magnetic resonance imaging in dementia. A comparative study. 7 Neurol Neurosurg Psychiatry 2000;69:630-5.

104 Frisoni GB, Geroldi C, Beltramello A, et al. Radial width of the temporal horn: a simple CT-based measure of medial temporal lobe atrophy sensitive to Alzheimer's disease. Neurology 2000;54(suppl 3):A107.

105 Gearing M, Mirra SS, Hedreen JC, et al. The Consortium to Establish a Registry for Alzheimer's Disease (CERAD). Part X. Neuropathology confirmation of the clinical diagnosis of Alzheimer's disease. Neurology 1995;45:461-6.

106 Smith AD, Jobst KA, Johnston C, et al. Apolipoprotein-E genotyping in diagnosis of Alzheimer's disease. Lancet 1996;348:483-4.

107 Lopez OL, Becker JT, Klunk W, et al. Research evaluation and diagnosis of probable Alzheimer's disease over the last two decades: I. Neurology 2000; 55:1854-62.

108 DeCarli C, Maisog J, Murphy DG, et al. Method for quantification of brain, ventricular, and subarachnoid CSF volumes from MR images. F Comput Assist Tomogr 1992;16:274-84.

109 Thompson P, Mega A, Toga A. Disease based atlases. In: Mazziotta J, Toga A, Frackowiak R, eds. Brain mapping: the disorders. San Diego, CA: Academic Press, 2000:131-77.

110 Scheltens P, Korf ES. Contribution of neuroimaging in the diagnosis of Alzheimer's disease and other dementias. Curr Opin Neurol 2000;13:391-6.

11 Small GW, Huang S-C, Cole G, et al. In vivo visualization of amyloid senile plaques and neurofibrillary tangles in Alzheimer's disease. Proceedings of the can Geophysical Union, 8 July 2000

112 Zhen W, Han H, Anguiano M, et al. Synthesis and amyloid binding properties of rhenium complexes: preliminary progress toward a reagent for 15 .

113 Skovronsky DM, Zhang B, Kung MP, et al. In vivo detection of amyloid plaques in a mouse model of Alzheimer's disease. Proc Natl Acad Sci USA 2000;97:7609-14.

114 Bookheimer SY, Strojwas MH, Cohen MS, et al. Patterns of brain activation in people at risk for Alzheimer's disease. N Engl F Med 2000;343: $450-6$.

115 Foster GR, Scott DA, Payne S. The use of CT scanning in dementia. A systematic review. Int $\mathcal{F}$ Technol Assess Health Care 1999;15:406-23.

116 Neumann PJ, Hermann RC, Kuntz KM, et al. Cost-effectiveness of donepezil in the treatment of mild or moderate Alzheimer's disease. Neurology 1999;52:1138-45.

117 Kenen RH. The at risk health status and technology: a diagnostic invitation and the gift of knowing. Soc Sci Med 1996;42:1545-53.

118 Prichard JW, Brass LM. New anatomical and functional imaging methods. Ann Neurol 1992;32:395-400. 